\title{
Monitorizando el consumo de cannabis en España a través de las aguas residuales en el marco de la red ESAR-Net
}

Rodil, Rosarioa, González-Mariño, Iriaa,b, Montes, Rosaa, Estévez-Danta, Andreaa, Hernández, Félixc, Celma, Albertoc, Bijlsma, Lubertusc, Picó, Yolandad, Andreu, Vicented, Álvarez, Rodrigod, López de Alda, Mirene, Postigo, Cristinae, López, Estere, Valcárcel, Yolandaf, Rico, Andreug, Pocurrull, Evah, Marcé, Rosa Mariah, Miró, Manueli, Prieto, Ailettej, Quintana, José Benitoa a Universidade de Santiago de Compostela, España, rosario.rodil@us.es, b Universidad de Salamanca, España, c Universitat Jaume I, España, d Universitat de Valencia/CSIC, España, e Instituto de Diagnóstico Ambiental y Estudios del Agua (IDAEA) del CSIC, España, f Universidad Rey Juan Carlos, España, g IMDEA Agua, España, h Universitat Rovira i Virgili, España, i Universitat de les Illes Balears, España, j Universidad del País Vasco, España.

\section{Introducción}

El análisis de aguas residuales para la determinación de productos de excreción metabólica humana (Análisis de Aguas Residuales con fines epidemiológicos) es una poderosa herramienta complementaria a los estudios socio-epidemiológicos tradicionales para estimar el abuso de sustancias en una localidad. Basada en el innovador concepto de que el agua residual equivale a una muestra de orina diluida e integrada de toda una comunidad, se ha utilizado para evaluar el consumo de drogas en diferentes países y ha recibido la consideración y atención de organismos internacionales como la Oficina de las Naciones Unidas contra la Droga y el Delito (UNODC) o el Observatorio Europeo de las Drogas y las Toxicomanías (EMCDDA). En el caso particular del cannabis, el aislamiento de su principal metabolito urinario, el 11-nor-9-carboxi- $\Delta 9$ tetrahidrocanabinol (THCCOOH), en las aguas conlleva un desafío analítico especial. Sin embargo, una vez desarrollado y validado el método de análisis, su aplicación permite cuantificar el consumo de $\Delta 9$-tetrahidrocanabinol (THC) en una localidad de forma objetiva, barata y en tiempo real. Esta metodología ya se ha empleado, por ejemplo en el estado de Washington (EEUU) para evaluar el impacto de la legalización del cannabis con carácter recreativo.

\section{Métodos}

Se tomaron muestras de agua residual integrada ( 24 horas) durante 2018 en 17 depuradoras de agua residual durante una semana en el marco de la red ESAR-Net (www.esarnet.es). Tras filtrar el agua, el THCCOOH se extrajo mediante un proceso de extracción en fase sólida y los extractos se analizaron mediante cromatografía de líquidos acoplada a espectrometría de masas en tándem (para determinar la concentración del mismo en la muestra). Finalmente, con los datos de concentración, volumen de agua residual procesado diariamente en la depuradora, y población servida por la misma, se procedió a estimar el consumo de THC en mg/(día.1000 hab). Además, para algunas ciudades españolas (aprox. 4) dicho ejercicio se lleva haciendo anualmente desde 2011.

\section{Resultados}

El análisis de los datos de consumo estimado de THC permitió detectar diferencias entre las diferentes ciudades. Además, también se han empleado estos resultados para extrapolar el consumo global de cannabis por la población española y compararlos con el consumo de otras sustancias. En esta presentación se presenta la metodología y los resultados obtenidos, considerando las ventajas e inconvenientes que puede aportar esta metodología en contraste con otros indicadores epidemiológicos y cuales son las dificultades en el caso del cannabis.

\section{Agradecimientos}

Consellería de Cultura, Educación e Ordenación Universitaria, Plan Galego I2C (programa postdoctoral, Modalidad B y ED431C2017/36). Agencia Estatal de Investigación (CTM2016-81935REDT y CTM2014-56628-C3-2-R) y FEDER. 